\title{
Voting Systems as Linear Transformation Models
}

\author{
M.A. El-Tawil*
}

Department of Engineering Mathematics, Faculty of Engineering, Cairo University, Giza, Egypt

\begin{abstract}
In this paper, a new voting system model is introduced as a linear transformation model. The size of compartmental populations in each political group corresponding to every voted subject, the population weights, the weighted votes policy against each political group, the "Yes" and "No" rates, the "No" rejection policy and the "Yes" increase policy are the inputs / outputs of the introduced linear models. Some parametric studies have been achieved concerning the answer of some interesting questions, for example: the effect of unfair election policy, the critical population size of the opposite political group and others. The inverse problem and a stochastic case are also introduced.
\end{abstract}

Keywords: Voting system, linear transformation, matrices.

\section{INTRODUCTION}

Generally, in most of the third word nations the elections are subjected to false policies from the government that lead to maximize required votes and minimize unwanted ones. In one of these countries, the government forbids the opposite nominees from completing their official papers and construct very long queues of pre-paid persons to prevent the other nominees who succeeded in getting completing official papers from recording their names in the short proper time. The election process itself is full of very bad events from artificial quarrelling among voters to faking or unfair votes. In some countries, the policy may lead to killing or imprisoning the opposites. The goal of all these policies is to transform a certain unwanted state, from governmental opinion, to a required state. In this paper, a linear transformation model is constructed to describe the voting system as a whole and allows such processes to be analyzed and controlled.

Formally, a voting system specifies the form of a ballot together with a tallying method. Since the works of JeanCharles de Borda and Marquis de Condorcet, the voting systems attracted the attention of many authors in different scientific fields. Many scientific thoughts have been applied in voting systems, for example: weighting votes, ranking votes, running off votes, single winning, multi winnings, proportionality and others, see [1-9]. In this paper, a voting system is introduced as a linear transformation in which the weighted populations of every political group of voters are the elements of the transformation matrix. The corresponding board policies are the elements of the policy vector which is transformed into the reference mass vector to which the "Yes" or "No" rates are related. In sections 2 to 6 ,deterministic models are described and tested in different case studies, one political group (section 3), two political groups (section 4), three political groups (section 5) and two voted subjects in section 6 . The inverse problem is introdu-

*Address correspondence to this author at the Department of Engineering Mathematics, Faculty of Engineering, Cairo University, Giza, Egypt; Tel: 0020101368172; Fax: 00202-35723486;

E-mail: magdyeltawil@yahoo.com ced in section 7 . In section 8 , a primitive stochastic or random model is introduced through a case study analyzing three possibilities concerning the "Yes" voters of a political group, mainly uniform, fall and rise cases.

\section{THE GENERAL DETERMINISTIC MODEL}

Let us assume that the voters of the $i^{\text {th }}$ political group in certain community are divided into three populations, the first $\beta_{i 1}^{(k)}$ is the "yes" voters, the second $\beta_{i 2}^{(k)}$ is the "no" voters and $\beta_{i 3}^{(k)}$ is the "neutral" voters who do not select neither "yes" nor "no", if this is allowed, may be "false" voting. If the total interested voters in the $i^{\text {th }}$ political group on the $k^{\text {th }}$ voted subject, for multi voted subjects, are $N_{i}^{(k)}$, then the following relation for $\mathrm{n}$ political groups must hold:

$\sum_{l=1}^{3} \beta_{i 1}^{(k)}=N_{i}^{(k)}, \quad i=1,2, \cdots n$.

Also, we must have the following relationship concerning the whole political system:

$\sum_{i=1}^{n} N_{i}^{(k)}=N^{(k)}, \quad k=1,2, \cdots m$,

where $N^{(k)}$ is the total voters on the $k^{\text {th }}$ voted subject.

Let us define the population weight of the $i^{\text {th }}$ political group on the $k^{\text {th }}$ voted subject as

$\alpha_{i}^{(k)}=\frac{N_{i}^{(k)}}{N^{(k)}}, \quad i=1,2, \cdots, n$

for which the following relationship must be satisfied

$\sum_{i=1}^{n} \alpha_{i}^{(k)}=1, \quad k=1,2, \cdots, m$

The action against political groups may be "fair" in fair voting or "unfair" in an unfair voting, may have different 
meanings in voting systems. These actions can be modeled when designing the action or policy parameters $l_{i j}^{(k)}, j=1,2,3$, where

i) $\mathrm{j}=1$ considers the "Yes" mass voters,

ii) $\mathrm{j}=2$ considers the "No" mass and

iii) $\mathrm{j}=3$ considers the "Neutral" mass.

The multiplication $\beta_{i 1}^{(k)} l_{i 1}^{(k)}$ represents the official "yes" voters after the action. If $l_{i 1}^{(k)}=1$, the voting system is fair, may have different terminology according to the voting system. If $l_{i 1}^{(k)}>1$, the voting system may give over weights for the "yes" voters, may be unfair. If $l_{i 1}^{(k)}<1$, the voting system may give under weights for the "yes" voters, may be unfair. The other actions are similar. Accordingly, an effective decisive population number $D_{i}^{(k)}$ can be evaluated as

$\sum_{j=1}^{3} \beta_{i j}^{(k)} l_{i j}^{(k)}=D_{i}^{(k)}, \quad i=1,2, \cdots, n$

which will be weighted by the population weight $\alpha_{i}^{(k)}$.

Now, we can evaluate the "Yes", "No" and "Neutral" ratios as follows:

$$
\begin{gathered}
R_{\text {yes }}^{(k)}=\frac{\sum_{i=1}^{n} \alpha_{i}^{(k)} \beta_{i 1}^{(k)} l_{i 1}^{(k)}}{D^{(k)}}, k=1,2, \ldots \quad m, \\
R_{\text {No }}^{(k)}=\frac{\sum_{i=1}^{n} \alpha_{i}^{(k)} \beta_{i 2}^{(k)} l_{i 2}^{(k)}}{D^{(k)}}, k=1,2, \ldots \quad m, \\
R_{\text {neutral }}^{(k)}=\frac{\sum_{i=1}^{n} \alpha_{i}^{(k)} \beta_{i 3}^{(k)} l_{i 3}^{(k)}}{D^{(k)}}, k=1,2, \ldots \quad m,
\end{gathered}
$$

where

$$
D^{(k)}=\sum_{i=1}^{n} \alpha_{i}^{(k)} \sum_{j=1}^{3} \beta_{i j}^{(k)} l_{i j}^{(k)}, k=1,2, \ldots \quad m .
$$

All these parameters can be reserved in a linear transformation model where all quantities are considered as deterministic ones. No uncertainties are considered in this section. The general model can be illustrated as follows:

$$
[\underbrace{A_{1}}_{\alpha_{1}^{(k)}} \underbrace{A_{2}}_{\alpha_{2}^{(k)}} \cdots \cdots \underbrace{A_{n}}_{\alpha_{n}^{(k)}}]_{m, 3 n}\left[\begin{array}{c}
L_{1} \\
L_{2} \\
\vdots \\
\vdots \\
L_{n}
\end{array}\right]_{3,1}=[D]_{m, 1}, k=1,2, \cdots, m
$$

where $A_{i}$ : the population information of the voters of the $\mathrm{i}$ th political group on $\mathrm{k}$-th voted subject,

$L_{i}$ : a sub-vector contains weighted votes policy against the i-th political group,

$D$ : the total effective decisive population numbers vector,

$\mathrm{n}$ : the number of the political groups,

$\mathrm{m}$ : the number of the voted subjects.

The elements of each row in $A_{i}$ represent the compartmental populations in the i-th group distributed on a voted subject w.r.t "Yes", "No" and "Neutral". The elements of the sub-vectors $L_{i}$ represent the election board policy (may be the government)or the action against the $\mathrm{i}$-th political group. The $D$ vector contains the effective decisive population numbers of the voted subjects for which "Yes", "No" and "Neutral" rates are computed in relation to these decisive numbers.

\section{The Model Rules and Outcomes}

The rules and outcomes of the model can be introduced as follows:

1. The model deals with $\mathrm{n}$ political groups (collations, parties, clubs ..etc) each has an $N_{i}^{(k)}$ population from the total allowed votes $N^{(k)}$. Accordingly, the ratios $\alpha_{i}^{(k)}=\frac{N_{i}^{(k)}}{N^{(k)}}, \quad i=1,2, \cdots, n$ representing the population weights of i-th political group in a political society, where $\sum_{i=1}^{n} \alpha_{i}^{(k)}=1, \quad k=1,2, \cdots, m$.

2. The compartmental populations of the $\mathrm{i}$-th group are $\beta_{i j}^{(k)}, j=1,2,3$ distributed on "Yes", No" and "Neutral" respectively concerning the voted subject $\mathrm{k}$, where

i) $\quad \mathrm{j}=1$ represents the "Yes" mass,

ii) $\quad \mathrm{j}=2$ represents the "No" mass and

iii) $j=3$ represents the "Neutral" mass.

The compartmental populations should satisfy the following condition:

$\sum_{j=1}^{3} \beta_{i j}^{(k)}=N_{i}^{(k)} \forall i, k$

3. When dividing $\beta_{i j}^{(k)}$ on $N_{i}^{(k)}$, one can get the compartmental population weights of the i-th political group for each voted subject $k$, i.e.

$\omega_{i j}^{(k)}=\frac{\beta_{i j}^{(k)}}{N_{i}^{(k)}}, j=1,2,3, \quad k=1,2,3, \ldots \quad m$.

The following condition must be satisfied:

$\sum_{j=1}^{3} \omega_{i j}^{(k)}=1 \forall i, k$. 
4. The election board policy against the $\mathrm{i}$-th political group can be represented as the policy numbers $l_{i j}, j=1,2,3$ where

i) $\quad \mathrm{j}=1$ represents the policy against "Yes" mass,

ii) $\quad j=2$ represents the policy against "No" mass and

iii) $\mathrm{j}=3$ represents the policy against "Neutral" mass.

For the most of the cases, $l_{i j}=1$ but reported different values may exist for some policies (fair, unfair or others). The system may be biased to "Yes" by taking $l_{i 1} \geq 1$, $l_{i 2}<1$ which maximizes "Yes" and minimizes "No". Different opposite view leads to a biased system to "No".

5. The effective decisive population number for the k-th voted subject is computed, using the model, as

$$
D^{(k)}=\sum_{i=1}^{n} \alpha_{i}^{(k)} \sum_{j=1}^{3} \beta_{i j}^{(k)} l_{i j}^{(k)}, k=1,2, \ldots \quad m
$$

for $\mathrm{m}$ independent voted subjects (for example, m-winners case).

6. The "Yes" rate of the k-th voted subject can now be computed as follows:

$$
R_{\text {yes }}^{(k)}=\frac{\sum_{i=1}^{n} \alpha_{i}^{(k)} \beta_{i 1}^{(k)} l_{i 1}^{(k)}}{D^{(k)}}, k=1,2, \ldots \quad m .
$$

7. Similarly, the "No" and "Neutral" rates are computed as follows:

$$
\begin{gathered}
R_{N o}^{(k)}=\frac{\sum_{i=1}^{n} \alpha_{i}^{(k)} \beta_{i 2}^{(k)} l_{i 2}^{(k)}}{D^{(k)}}, k=1,2, \ldots \quad m, \\
R_{\text {neutral }}^{(k)}=\frac{\sum_{i=1}^{n} \alpha_{i}^{(k)} \beta_{i 3}^{(k)} l_{i 3}^{(k)}}{D^{(k)}}, k=1,2, \ldots \quad m .
\end{gathered}
$$

It is so easy to show that

$$
R_{\text {yes }}^{(k)}+R_{n o}^{(k)}+R_{\text {neutral }}^{(k)}=1, \quad k=1,2, \ldots \quad m .
$$

8. As a special case, considering equally likely population ratios for all the political groups

( $\alpha_{i}=\frac{1}{n}$ ), the following simpler relations are got:

$$
\begin{gathered}
R_{y e s}^{(k)}=\frac{\sum_{i=1}^{n} \omega_{i 1}^{(k)} l_{i 1}^{(k)}}{\sum_{i=1}^{n} \sum_{j=1}^{3} \omega_{i j}^{(k)} l_{i j}^{(k)}}, k=1,2, \ldots \quad m, \\
R_{n o}^{(k)}=\frac{\sum_{i=1}^{n} \omega_{i 2}^{(k)} l_{i 2}^{(k)}}{\sum_{i=1}^{n} \sum_{j=1}^{3} \omega_{i j}^{(k)} l_{i j}^{(k)}}, k=1,2, \ldots \quad m,
\end{gathered}
$$

$$
R_{\text {yes }}^{(k)}=\frac{\sum_{i=1}^{n} \omega_{i 3}^{(k)} l_{i 3}^{(k)}}{\sum_{i=1}^{n} \sum_{j=1}^{3} \omega_{i j}^{(k)} l_{i j}^{(k)}}, k=1,2, \ldots \quad m .
$$

9. "No" rejection policy, voting systems biased toward "Yes" decision

In this policy, the policy parameters take the following values:

$$
l_{i 1}=1, l_{i 2}=\beta, l_{i 3}=0,0 \leq \beta \leq 1, i=1,2, \cdots, n .
$$

This means that we reject $(1-\beta)$ of the "No" votes. In this case, the "Yes" rate is

$$
\begin{aligned}
R_{y e s}^{(k)} & =\frac{\sum_{i=1}^{n} \alpha_{i}^{(k)} \omega_{i 1}^{(k)}}{\sum_{i=1}^{n} \alpha_{i}^{(k)} \sum_{j=1}^{3} \omega_{i j}^{(k)} l_{i j}^{(k)}}, k=1,2, \ldots \quad m \\
& =\frac{\sum_{i=1}^{n} \alpha_{i}^{(k)} \omega_{i 1}^{(k)}}{\sum_{i=1}^{n} \alpha_{i}^{(k)} \omega_{i 1}^{(k)}+\beta \sum_{i=1}^{n} \alpha_{i}^{(k)} \omega_{i 2}^{(k)}}
\end{aligned}
$$

The system may be considered a "fair voting system" when $\beta=1$, otherwise it is "unfair voting system ", or according to the voting system terminology.

10. "Yes" increase policy, voting systems biased toward "Yes" decision

In this policy, the policy parameters take the following values:

$l_{i 1}=1+\alpha, l_{i 2}=1, l_{i 3}=0, \alpha \geq 0, i=1,2, \cdots, n$.

This means that we put extra weights on "Yes" votes or it may carry another bad meaning, a fake voting system. In this case, the "Yes" rate is

$$
\begin{aligned}
R_{y e s}^{(k)} & =\frac{\sum_{i=1}^{n} \alpha_{i}^{(k)} \omega_{i 1}^{(k)}(1+\alpha)}{\sum_{i=1}^{n} \alpha_{i}^{(k)} \sum_{j=1}^{3} \omega_{i j}^{(k)} l_{i j}^{(k)}}, k=1,2, \ldots \quad m \\
& =\frac{\sum_{i=1}^{n} \alpha_{i}^{(k)} \omega_{i 1}^{(k)}(1+\alpha)}{\sum_{i=1}^{n} \alpha_{i}^{(k)} \omega_{i 1}^{(k)}(1+\alpha)+\sum_{i=1}^{n} \alpha_{i}^{(k)} \omega_{i 2}^{(k)}}
\end{aligned}
$$

The system may be considered a "fair voting system" when $\alpha=0$, otherwise it is "unfair voting system ".

\section{Important property}

Concerning the "No" rejection and the "Yes" increase policies, the value $\beta=\frac{1}{1+\alpha}$ makes $\alpha$ and $\beta$ produce the same "Yes" rate. 


\section{Proof}

This means that we have

$$
\frac{\sum_{i=1}^{n} \alpha_{i}^{(k)} \omega_{i 1}^{(k)}(1+\alpha)}{\sum_{i=1}^{n} \alpha_{i}^{(k)} \omega_{i 1}^{(k)}(1+\alpha)+\sum_{i=1}^{n} \alpha_{i}^{(k)} \omega_{i 2}^{(k)}}=\frac{\sum_{i=1}^{n} \alpha_{i}^{(k)} \omega_{i 1}^{(k)}}{\sum_{i=1}^{n} \alpha_{i}^{(k)} \omega_{i 1}^{(k)}+\sum_{i=1}^{n} \alpha_{i}^{(k)} \omega_{i 2}^{(k)} \beta} .
$$

Dividing the 1.h.s. of the equation by the factor $(1+\alpha)$ proves the statement.

This means that the two policies are equivalent in the sense that they produce the same "Yes" rate.

\section{ONE POLITICAL GROUP MODEL}

In this case, a simple model can be illustrated as follows, $\mathrm{k}=1$ is dropped:

$\left[\begin{array}{lll}\beta_{11} & \beta_{12} & \beta_{13}\end{array}\right]\left[\begin{array}{l}l_{11} \\ l_{12} \\ l_{13}\end{array}\right]=[D]$.

Let us examine the model through the following notes:

$N-1 \quad$ Taking the policy parameters as follows:

$l_{11}=1, l_{12}=0, l_{13}=0$,

the "Yes" rate is

$$
R_{y e s}^{(1)}=\frac{\omega_{11}(1)}{\omega_{11}(1)+\omega_{12}(0)+\omega_{13}(0)}=100 \% .
$$

This is the case of a total "Yes" system irrespective of the compartmental population weights.

$N-2$ If the policy is changed to take $l_{11}=0, l_{12}=1, l_{13}=0$, the system is transferred to a total "No" system with $R_{n o}^{(1)}=100 \%$.

$N-3$ For the case of a "fair voting system", or no weights voting cases, the policy is changed to $l_{11}=1, l_{12}=1, l_{13}=0$ which means that the voting system considers all the "yes" votes, all the "No" votes and none of the neutrals (false votes) without any interference. In this case,

$R_{\text {yes }}^{(1)}=\frac{\omega_{11}}{\omega_{11}+\omega_{12}}$.

$N$-4) For the case of an "unfair voting system", or alike, and taking the "No rejection policy" which reduces the "No" votes, the policy parameters are

$l_{11}=1, l_{12}=\beta<1, l_{13}=0$.

In this case

$\left.R_{\text {yes }}^{(1)}\right|_{\text {unfair }}=\frac{\omega_{11}}{\omega_{11}+\beta \omega_{12}}>\left.R_{\text {yes }}^{(1)}\right|_{\text {fair }}$.
Fig. (1) illustrates the change of the "Yes" rate with $\beta$.

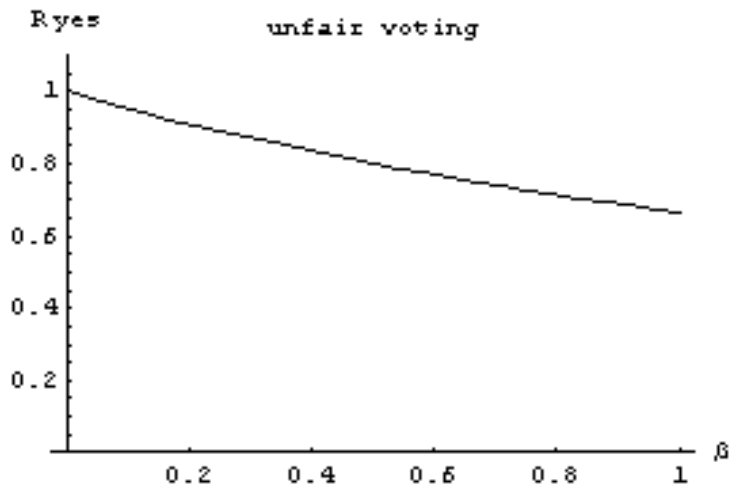

Fig. (1). The change of the "Yes" rate with $\beta$ in the case of "No rejection policy".

One can notice that $\beta=1$ is reduced to "fair voting" while $\beta=0$ is the case of a total "Yes" system.

$N-5) \quad$ For the case of "unfair voting system", or alike, and taking the policy of increasing "Yes" votes, the policy numbers are $l_{11}=1+\alpha, l_{12}=1, l_{13}=0, \quad \alpha>0$.

In this case

$$
\begin{aligned}
\left.R_{\text {yes }}^{(1)}\right|_{\text {uffair }} & =\frac{\omega_{11}(1+\alpha)}{\omega_{11}(1+\alpha)+\omega_{12}} \\
& =\frac{\omega_{11}+\alpha \omega_{11}}{\omega_{11}+\omega_{12}+\alpha \omega_{11}} \\
& =\frac{\omega_{11}}{\omega_{11}+\omega_{12}}\left[\frac{(1+\alpha)}{\left(1+\alpha \frac{\omega_{12}}{\omega_{11}+\omega_{12}}\right)}\right] \\
& =\left.\left[\frac{(1+\alpha)}{\left(1+\alpha \frac{\omega_{12}}{\omega_{11}+\omega_{12}}\right)}\right] R_{\text {yes }}^{(1)}\right|_{\text {fair }} \\
& >\left.R_{\text {yes }}^{(1)}\right|_{\text {fair }}
\end{aligned}
$$

\section{TWO POLITICAL GROUPS MODELS}

The model takes the following form, $\mathrm{k}=1$ is dropped:

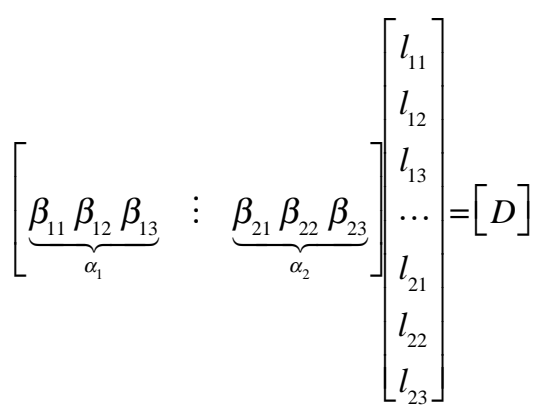

where $\beta_{i 1}+\beta_{i 2}+\beta_{i 3}=N_{i}, i=1,2$ and $\alpha_{1}+\alpha_{2}=1$. The following notes can be considered:

$N-1) \quad$ This system represents two political groups, the first may be the governmental group while the other may 
represent the opposite one. They are voting on a one voted subject, say the new president or whatever.

$N$-2) Let us have a fair policy, or alike, say $l_{11}=1, l_{12}=1, l_{13}=0$ and

$l_{21}=1, l_{22}=1, l_{23}=0$. In this case

$R_{y e s}^{(1)}=\frac{\alpha_{1} \omega_{11}+\alpha_{2} \omega_{22}}{\left(\omega_{11}+\omega_{12}\right) \alpha_{1}+\left(\omega_{21}+\omega_{22}\right) \alpha_{2}}$.

For example taking $\omega_{11}=.8, \omega_{12}=.1, \omega_{13}=.1$, i.e. $80 \%$ of the governmental group vote "Yes" while $10 \%$ say "No" and the rest are neutral (false voting). The opposites may have a contradicting opinion as $\omega_{21}=.05, \omega_{22}=.9, \omega_{23}=.05$. If the relative population weight of the governmental group is $\alpha_{1}=\gamma$, then the corresponding weight for opposites is $\alpha_{2}=1-\gamma$. Accordingly, the "Yes" rate is

$$
\begin{aligned}
R_{y e s}^{(1)} & =\frac{.8 \alpha+.05(1-\gamma)}{(.9) \gamma+(.95)(1-\gamma)} \\
& =\frac{1+15 \gamma}{19-\gamma}, 0 \leq \gamma \leq 1
\end{aligned}
$$

Fig. (2) illustrates the change of the "Yes" rate with the population weight $\gamma$.

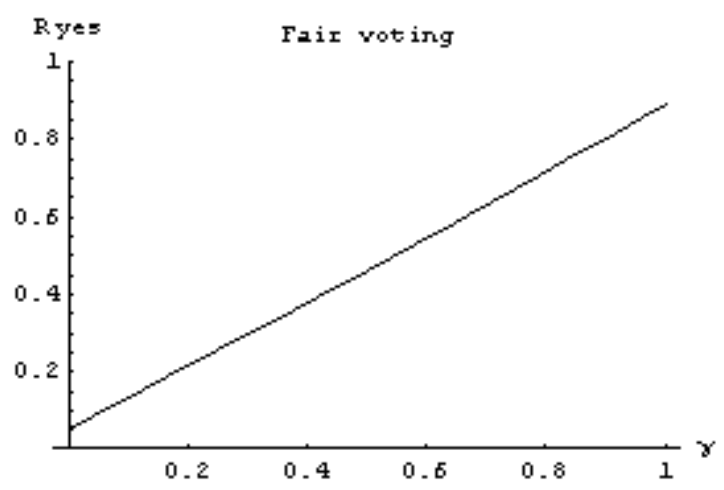

Fig. (2). The change of the "Yes" rate with the governmental population weight $\gamma$.

One can notice the high and logical effect of $\gamma$ on the "Yes" rate, the increase of $\gamma$ causes the increase of the rate. To insure that $R_{y e s}^{(1)}>.5$, we should have $\gamma>54.84 \%$. This has a very important meaning for the governmental group to have "Yes" on their voted subject. Oppositely, the opposite group should be maintained beyond $45.16 \%$ of the voting populations to keep the "Yes" possibility. Otherwise, the opposites will win.
$N$-3) Let us take the case of an "unfair voting" by taking the following action against opposite group $l_{21}=1, l_{22}=\beta<0, l_{23}=0$. In this case

$$
\left.R_{\text {yes }}^{(1)}\right|_{\text {unfair }}=\frac{\gamma \omega_{11}+(1-\gamma) \omega_{22}}{\left(\omega_{11}+\omega_{12}\right) \gamma+\left(\omega_{21}+\beta \omega_{22}\right)(1-\gamma)}>\left.R_{\text {yes }}^{(1)}\right|_{\text {fair }}
$$

since $\beta \leq 1$ and all the quantities are positive. Using the example in $(N-2)$, one can get

$$
\begin{aligned}
R_{y e s}^{(1)} & =\frac{.8 \gamma+.05(1-\gamma)}{(.9) \gamma+(.05+.9 \beta)(1-\gamma)} \\
& =\frac{1+15 \gamma}{1+18 \beta+(17-18 \beta) \gamma}, 0 \leq \gamma, \beta \leq 1
\end{aligned}
$$

Fig. (3) illustrates the global change of "Yes" rate with both $\beta$ and $\gamma$.

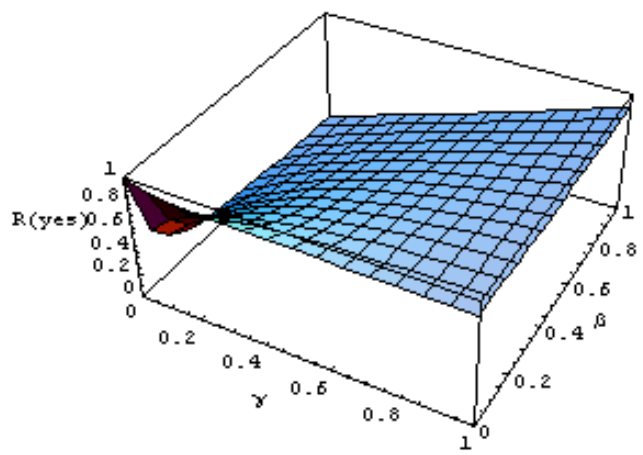

Fig. (3). The global change of the "Yes" rate with the weights $\beta$; the "No" policy parameter and $\gamma$; the governmental population weight.

It is clear that the high values of the parameters lead to the highest values of the rate. Fig. (4) illustrates the change of the rate with the different values of the parameters.

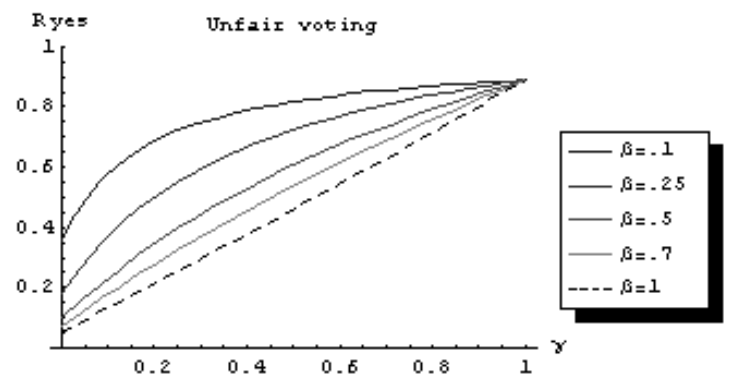

Fig. (4). The global change of the "Yes" rate with the weights $\beta$; the "No" policy parameter and $\gamma$; the governmental population weight.

Table 1. The Change of $\gamma$ with the Increase of $\beta$ to Insure $R_{y e s}^{(1)}>.5$

\begin{tabular}{|c|c|c|c|c|c|c|c|c|c|c|}
\hline $\boldsymbol{\beta}$ & $\mathbf{. 1}$ & $\mathbf{. 2}$ & $\mathbf{. 3}$ & $\mathbf{. 4}$ & $\mathbf{. 5}$ & $\mathbf{. 6}$ & $\mathbf{. 7}$ & $\mathbf{. 8}$ & $\mathbf{. 9}$ & $\mathbf{1 . 0}$ \\
\hline \hline$\% \gamma>$ & 5.4 & 15.66 & 23.91 & 30.69 & 36.36 & 41.18 & 45.31 & 48.91 & 52.07 & 54.84 \\
\hline
\end{tabular}


Under the condition that $R_{\text {yes }}^{(1)}>.5$, one can notice through the study of Table 1 that the decrease of $\beta$ removes the pressure on the governmental population weight $\gamma$.

$N$-4) Let us take another case of an "unfair voting" by taking the following action toward the governmental group $l_{11}=1+\alpha, l_{12}=1, l_{13}=0$. In this case

$$
\begin{aligned}
\left.R_{\text {yes }}^{(1)}\right|_{\text {unfair }} & =\frac{\gamma(1+\alpha) \omega_{11}+(1-\gamma) \omega_{22}}{\left((1+\alpha) \omega_{11}+\omega_{12}\right) \gamma+\left(\omega_{21}+\omega_{22}\right)(1-\gamma)} \\
& =\frac{\gamma \omega_{11}+(1-\gamma) \omega_{22}+\alpha \gamma \omega_{11}}{\left(\omega_{11}+\omega_{12}\right) \gamma+\left(\omega_{21}+\omega_{22}\right)(1-\gamma)+\alpha \gamma \omega_{11}} \\
& >\left.R_{\text {yes }}^{(1)}\right|_{\text {fair }}
\end{aligned}
$$

by using the fact that $\frac{n+h}{m+h}>\frac{n}{m}, h>0, n<m$.

Using the example in $(N-2)$, one can get

$$
\begin{aligned}
R_{y e s}^{(1)} & =\frac{.8 \gamma(1+\alpha)+.05(1-\gamma)}{(.8 \gamma(1+\alpha)+.1 \gamma)+.95(1-\gamma)} \\
& =\frac{.05+.75 \gamma+.8 \alpha \gamma}{.95-.05 \gamma+.8 \alpha \gamma} \\
& =\frac{1+15 \gamma+16 \alpha \gamma}{19-\gamma+16 \alpha \gamma}, \alpha>0,0 \leq \gamma \leq 1
\end{aligned}
$$

Fig. (5) illustrates the global change of "Yes" ratio with both $\gamma$ and $\alpha$.

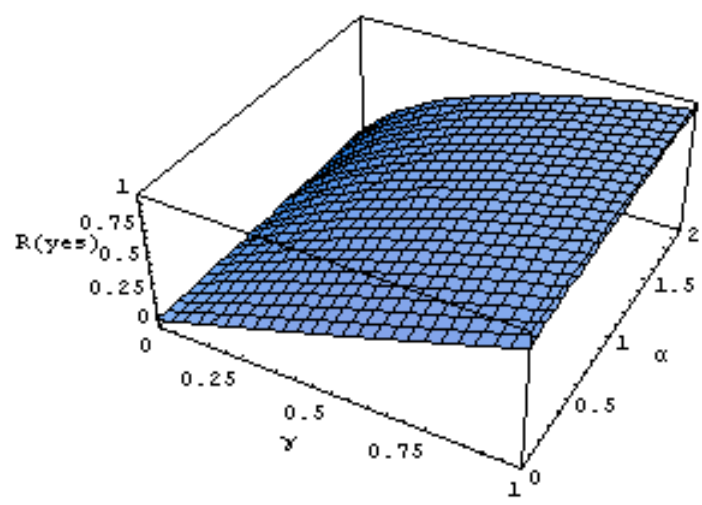

Fig. (5). The global change of "Yes" ratio with both $\gamma$ and $\alpha$.

Fig. (6) illustrates the change of the rate with the different values of the parameters. Under the condition that $R_{\text {yes }}^{(1)}>.5$, one can notice through the study of Table 2 that the

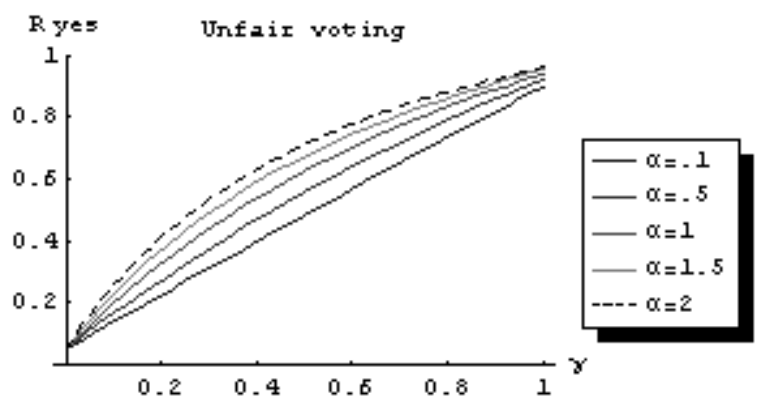

Fig. (6). The global change of the "Yes" rate with the weight $\alpha$, the "Yes" policy parameter and $\gamma$, the governmental population weight.

increase of $\alpha$ removes the pressure on the governmental population weight $\gamma$.

\section{THREE POLITICAL GROUPS MODELS}

The model takes the following form, $\mathrm{k}=1$ is dropped:

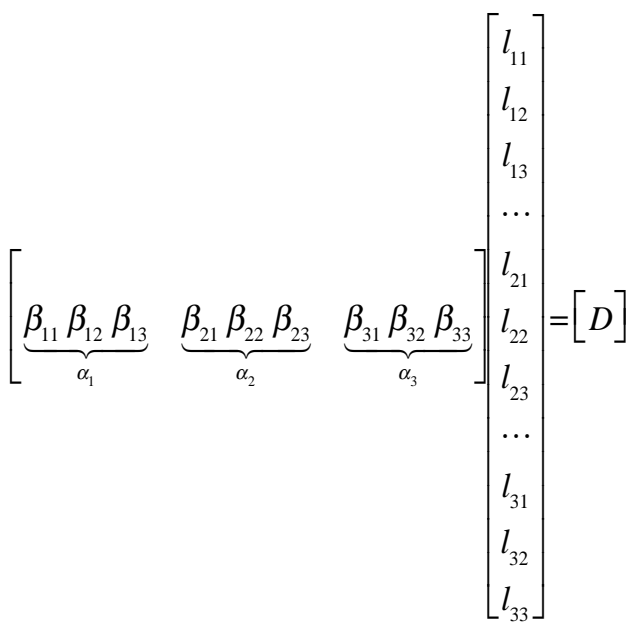

where $\beta_{i 1}+\beta_{i 2}+\beta_{i 3}=N_{i}, i=1,2,3$ and $\alpha_{1}+\alpha_{2}+\alpha_{3}=1$. The following notes can be considered.

$N$-1) This system may represent three political groups, the first is a governmental group, the second is an opposite group and an independent third one. They are voting on a one voted subject, say the new president or whatever.

$N$-2) Let us take a Fair policy, or alike, say $l_{11}=1, l_{12}=1$, $l_{13}=0, l_{21}=1, l_{22}=1, l_{23}=0$ and $l_{31}=1, l_{32}=1, l_{33}=0$. This means that a fair voting is established and no

Table 2. The Change of $\gamma$ with the Increase of $\alpha$ to Insure $R_{y e s}^{(1)}>.5$

\begin{tabular}{|c|c|c|c|c|c|c|}
\hline$\alpha$ & 0.0 & .2 & .4 & .6 & .8 & 38.81 \\
\hline$\% \gamma>$ & 54.84 & 49.71 & 45.46 & 41.87 & 2.0 \\
\hline$\alpha$ & 1.2 & 1.4 & 1.6 & 1.8 & 26.17 \\
\hline$\gamma>$ & 33.87 & 31.84 & 30.04 & 28.43 & 2.98 \\
\hline
\end{tabular}


rejection policies are considered from the voting board (usually governmental board). In this case

$R_{y e s}^{(1)}=\frac{\alpha_{1} \omega_{11}+\alpha_{2} \omega_{22}+\alpha_{3} \omega_{33}}{\left(\omega_{11}+\omega_{12}\right) \alpha_{1}+\left(\omega_{21}+\omega_{22}\right) \alpha_{2}+\left(\omega_{31}+\omega_{32}\right) \alpha_{3}}$

For example take $\omega_{11}=.8, \omega_{12}=.1, \omega_{13}=.1$ for the governmental group. The opposites may have a contradicting opinion as $\omega_{21}=.05, \omega_{22}=.9, \omega_{23}=.05$. Let the independent group has the following weights $\omega_{31}=.5, \omega_{32}=.4, \omega_{33}=.1$ Considering an unpopular governmental group, one can estimate its population rate as $\alpha_{1}=.2$. Let the population weight of the opposite group is $\alpha_{2}=\alpha$. Accordingly, the corresponding weight for the independence is $\alpha_{3}=.8-\alpha$. Accordingly, the "Yes" rate can be computed as

$$
\begin{aligned}
R_{y e s}^{(1)} & =\frac{.8(.2)+.05 \alpha+.5(.8-\alpha)}{(.9)(.2)+(.95) \alpha+.9(.8-\alpha)} \\
& =\frac{56-45 \alpha}{90+5 \alpha}, 0 \leq \alpha \leq .8
\end{aligned}
$$

Fig. (7) illustrates the change of the "Yes" rate with the population weight $\alpha$.

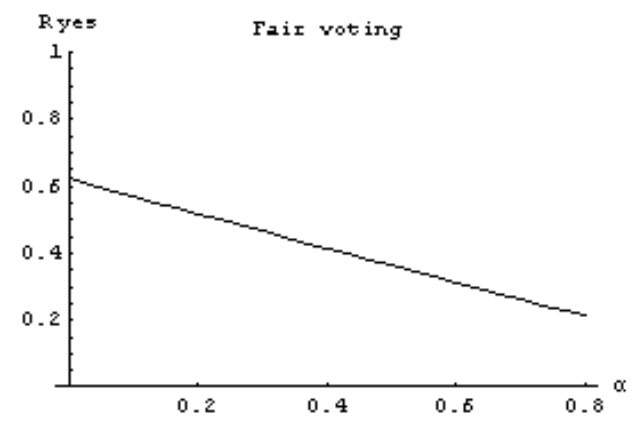

Fig. (7). The change of the "Yes" rate with the opposite population weight $\alpha$.

One can notice that if the independence group joins the opposite one, the result will be a disaster on the governmental group, corresponding to minimum "Yes" rate. The hope for such a government is keeping the opposites to a minimum weight to get around 55\% "Yes" rate which is a critical situation for a governing political group losing its popularity. In fact, this case finds a lot of examples in third word nations where the government can face this situation by taking false policies to survive.

Now, let us study the opposite group possibilities to win $\left(R_{\text {yes }}^{(1)}<.5\right)$. Let the maximum "Yes" ratio be $\mathrm{r}$, then we get the following inequality, $\frac{56-45 \alpha}{90+5 \alpha}<r$ which yields

$\alpha>\frac{56-90 r}{45+5 r}$.

For $0 \leq \alpha \leq .8$, we must have $.2128 \leq r \leq .622$. Fig. (8) illustrates the feasible region of $\alpha$.

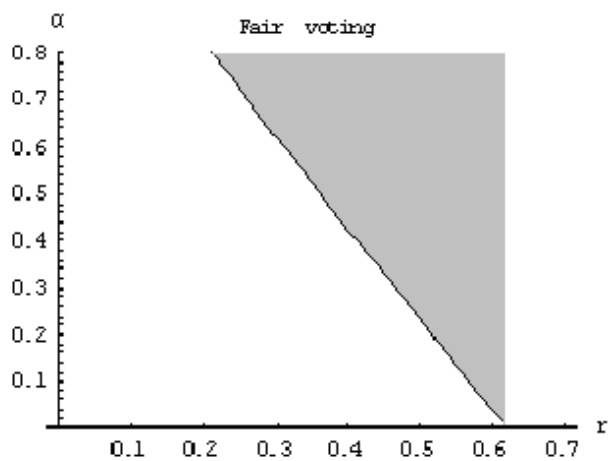

Fig. (8). The change of the population weight of the opposite group $\alpha$ with the maximum "Yes" rate.

The following table, Table $\mathbf{3}$, registers the minimum values of $\alpha$ versus the maximum "Yes" rate.

To decrease the "Yes" rate from .5 to .495 , the opposite group's popularity should increase from $23.16 \%$ to $24.12 \%$ (about $4 \%$ increase). To decrease the "Yes" rate from .5 to .4 , the popularity should increase from $23.16 \%$ to $42.55 \%$, i.e. $83.7 \%$ increase.

\section{Unfair Voting Case, "No" Rejection Policy}

As a reaction of an unpopular governmental political group, it may follow an unfair voting policy. Let it rejects $(1-\beta)$ of the "No" votes, i.e. $l_{i 1}=1, l_{i 2}=\beta, l_{i 3}=0, i=1,2,3$. In this case, the "Yes" rate is

$$
R_{y e s}^{(1)}=\frac{\omega_{11} \alpha_{1}+\omega_{21} \alpha_{2}+\omega_{31} \alpha_{3}}{\left(\omega_{11}+\omega_{12} \beta\right) \alpha_{1}+\left(\omega_{21}+\omega_{22} \beta\right) \alpha_{2}+\left(\omega_{31}+\omega_{32} \beta\right) \alpha_{3}} .
$$

Consider the case of having a saturated population weight $\alpha_{1}=s$ and a dynamic population weight $\alpha_{2}=\alpha$, then $\alpha_{3}=1-s-\alpha$. In this case, The "Yes rate becomes

$$
\begin{aligned}
& \left.R_{\text {yes }}^{(1)}\right|_{\text {lunfair }}=\frac{\omega_{11} s+\omega_{21} \alpha+\omega_{31}(1-s-\alpha)}{\left(\omega_{11}+\omega_{12} \beta\right) s+\left(\omega_{21}+\omega_{22} \beta\right) \alpha+\left(\omega_{31}+\omega_{32} \beta\right)(1-s-\alpha)} \\
& \quad=\frac{\omega_{11} s+\omega_{21} \alpha+\omega_{31}(1-s-\alpha)}{\left[\omega_{11} s+\omega_{21} \alpha+\omega_{31}(1-s-\alpha)\right]+\beta\left[\omega_{12} s+\omega_{22} \alpha+\omega_{32}(1-s-\alpha)\right]} \\
& >R_{\text {yes }\left.\right|_{\text {fair }} ^{(1)}}
\end{aligned}
$$

Table 3. The Change of the Minimum Values of $\alpha$ Versus the Maximum "Yes" Rate, Fair Voting

\begin{tabular}{|c|c|c|c|c|c|c|c|c|c|c|}
\hline $\mathrm{r}$ & .3 & .35 & .4 & .45 & .46 & .47 & .48 & .49 & .495 & .5 \\
\hline $\min \alpha$ & .623 & .524 & .42 & .32 & .30 & .28 & .27 & .25 & .24 & .23 \\
\hline
\end{tabular}




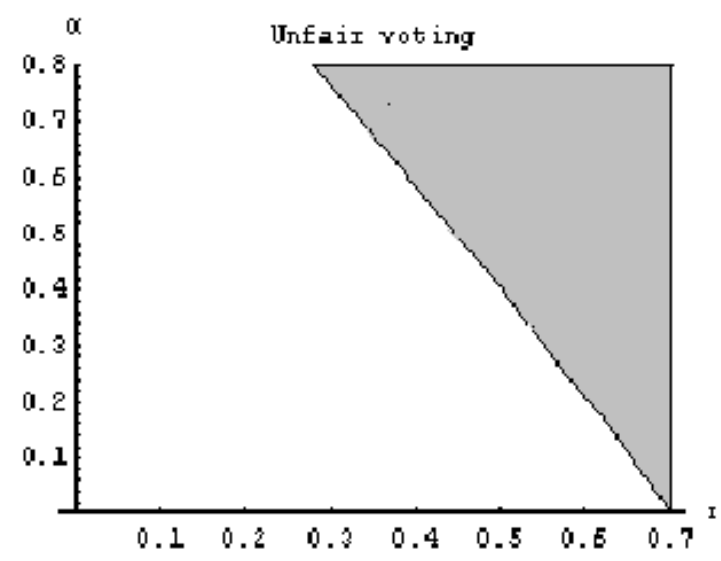

Fig. (9). The change of the population weight of the opposite group $\alpha$ with the maximum "Yes" rate.

From the opinion of the opposite political group, it is required to satisfy the following criteria

$\left.R_{\text {yes }}^{(1)}\right|_{\text {unfair }}<r, r \leq .5$

which leads to the following inequality

$\alpha \geq \frac{\omega_{11} s+\omega_{31}(1-s)-\left[\omega_{11} s+\omega_{31}(1-s)+\beta \omega_{12} s+\beta \omega_{32}(1-s)\right] r}{\left(\omega_{31}-\omega_{21}\right)+\left[\omega_{21}-\omega_{31}+\beta\left(\omega_{22}-\omega_{32}\right)\right] r}$

Three branches can be noticed here:

i) a straight line case in which

$\beta_{c}=\frac{\omega_{31}-\omega_{21}}{\omega_{22}-\omega_{32}}$

whereas

$\alpha \geq \frac{\omega_{11} s+\omega_{31}(1-s)-\left[\omega_{11} s+\omega_{31}(1-s)+\beta_{c} \omega_{12} s+\beta_{c} \omega_{32}(1-s)\right] r}{\left(\omega_{31}-\omega_{21}\right)}$,

$0 \leq \alpha \leq 1-s$

ii)

$\omega_{21}-\omega_{31}+\beta\left(\omega_{22}-\omega_{32}\right)>0$,

iii)

$$
\omega_{21}-\omega_{31}+\beta\left(\omega_{22}-\omega_{32}\right)<0 .
$$

Cases ii) and iii) are the cases of two different curvatures.

\section{Example-5.1.1}

Taking $\beta=.7$ and $\mathrm{s}=.2$ as the previous example. Considering the same weights, one can get the following relation for the "Yes" rate:

$$
\begin{aligned}
R_{y e s}^{(1)} & =\frac{.8(.2)+.05 \alpha+.5(.8-\alpha)}{(.87)(.2)+(.68) \alpha+.78(.8-\alpha)} \\
& =\frac{56-45 \alpha}{79.8-10 \alpha}, 0 \leq \alpha \leq .8
\end{aligned}
$$

To satisfy the criteria of having $\left.R_{\text {yes }}^{(1)}\right|_{\text {unfair }}<r, r \leq .5$, the population weight of the opposite group should finally satisfy

$\alpha>\frac{56-79.8 r}{45-10 r}$

For $0 \leq \alpha \leq .8$, we must have $.2786 \leq r \leq .7017$. Fig. (9) illustrates the feasible region of $\alpha$.

One can notice the change of curvature sign of the curve than that of Fig. (8). The following table, (Table 4), registers the minimum values of $\alpha$ versus the maximum "Yes" rate.

One can notice the high effect of the unfair policy of rejecting $30 \%$ of "No" votes. The popularity of the opposite group has been increased dramatically to do the same effect. For example, to insure that $R_{y e s}^{(1)}<.5$, the minimum $\alpha$ should be $40.25 \%$ in case of unfair voting while it is only $23.16 \%$ in fair voting case. This definitely helps a bad government group with low popularity to survive against honesty and fairness.

\section{Example- 5.1.2}

Consider the same previous example with taking a varying $\beta$ and $\mathrm{s}=.2$. In this case, the "Yes" rate is

$$
\begin{aligned}
R_{y e s}^{(1)} & =\frac{.8(.2)+.05 \alpha+.5(.8-\alpha)}{(.8+.1 \beta)(.2)+(.05+.9 \beta) \alpha+(.5+.4 \beta)(.8-\alpha)} \\
& =\frac{56-45 \alpha}{(56+34 \beta)+(50 \beta-45) \alpha}, 0 \leq \alpha \leq .8
\end{aligned}
$$

For a straight line case, $\beta_{c}=.9$, the following inequality is obtained:

$$
\begin{aligned}
\alpha & \geq \frac{\omega_{11} s+\omega_{31}(1-s)-\left[\omega_{11} s+\omega_{31}(1-s)+\beta_{c} \omega_{12} s+\beta_{c} \omega_{32}(1-s)\right] r}{\left(\omega_{31}-\omega_{21}\right)}, 0 \leq \alpha \leq 1-s \\
& =\frac{1}{.5-.05}[.8(.2)+.5(.8)-(.8(.2)+.5(.8)+.9(.1)(.2)+.9(.4)(.8)) r] \\
& =\frac{1}{45}[56-86.6 r]
\end{aligned}
$$

For $0 \leq \alpha \leq .8$, we must have $.2309 \leq r \leq .6467$. Fig. (10) illustrates the feasible region of $\alpha$.

Table 4. The Change of the Minimum Values of $\alpha$ Versus the Maximum "Yes" Rate, Unfair Voting: $\beta=.7$

\begin{tabular}{|c|c|c|c|c|c|c|c|c|c|c|}
\hline $\mathrm{r}$ & .3 & .35 & .4 & .45 & .46 & .47 & .48 & .49 & .495 & .5 \\
\hline $\min \alpha$ & .763 & .6764 & .5873 & .4961 & .4775 & .4589 & .4402 & .4214 & .4120 & .4025 \\
\hline
\end{tabular}




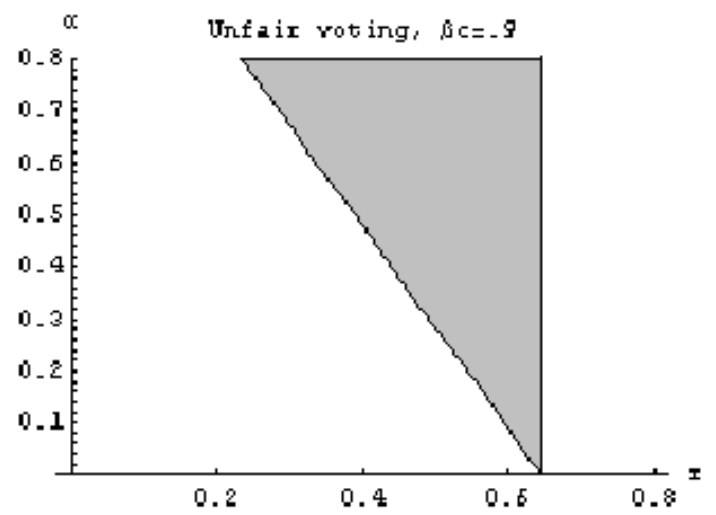

Fig. (10). The change of the population weight of the opposite group $\alpha$.

with the maximum "Yes" rate, $\beta=\beta_{c}=.9$.

Now let $\beta>.9$, then we get

$\alpha>\frac{56-(56+34 \beta) r}{45+(50 \beta-45) r}, .9<\beta<1$

Figs. $(\mathbf{1 1 , 1 2})$ illustrate the general behavior of $\alpha$ versus $\mathrm{r}$ and $\beta$.

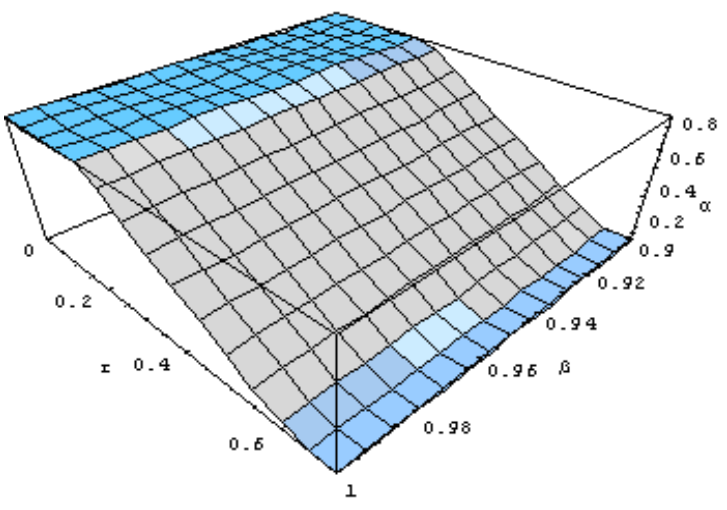

Fig. (11). The global change of $\alpha$ versus $\mathrm{r}$ and $\beta$.

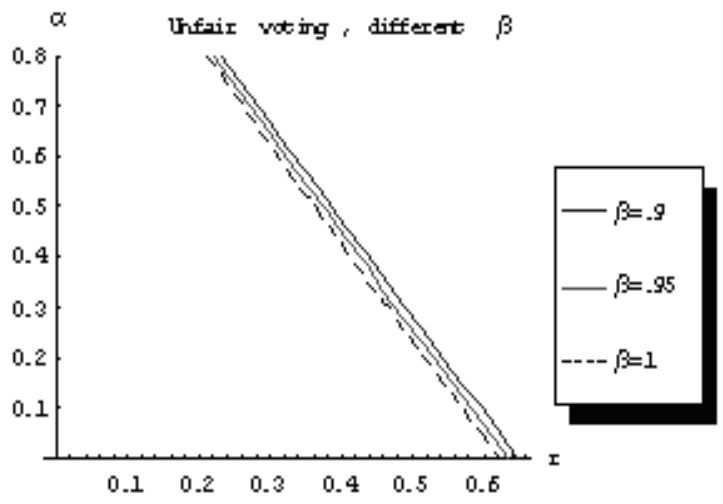

Fig. (12). The change of $\alpha$ versus $\mathrm{r}$ for diferent $\beta$.
For $0 \leq \alpha \leq .8$, we must have $\frac{20}{20+74 \beta}$ $\leq r \leq \frac{56}{56+34 \beta}, .9<\beta<1$.

One can notice that the fair voting case is approached when $\beta=1$.

Now let $\beta<.9$, then we get

$\alpha>\frac{56-(56+34 \beta) r}{45-(45-50 \beta) r}, 0<\beta<.9$

Figs. $(13,14)$ illustrate the general behavior of $\alpha$ versus $\mathrm{r}$ and $\beta$.

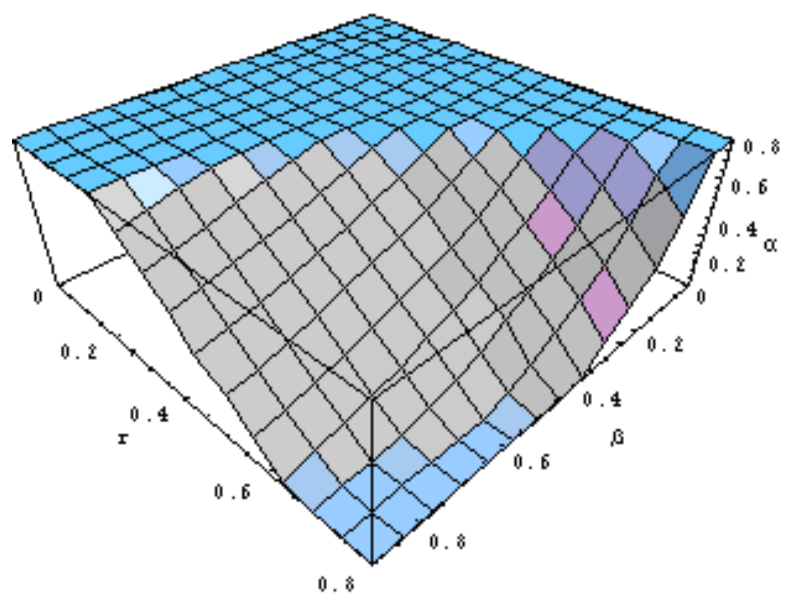

Fig. (13). The global change of $\alpha$ versus $\mathrm{r}$ and $\beta$.

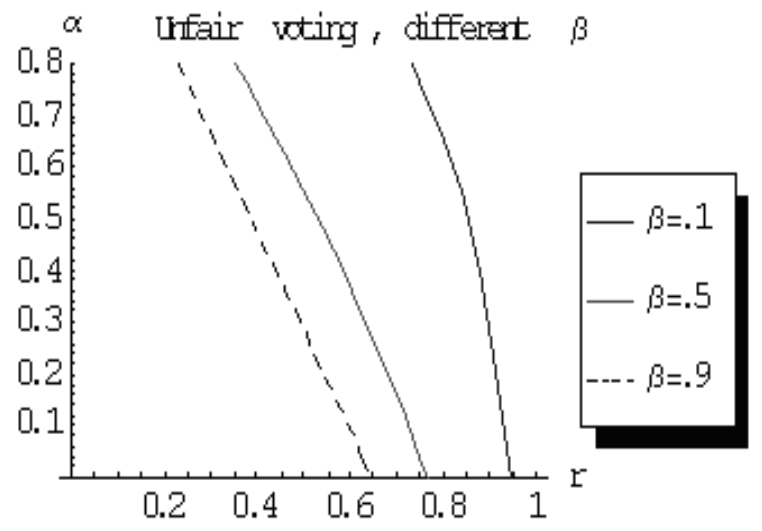

Fig. (14). The change of $\alpha$ versus $\mathrm{r}$ for different $\beta$.

For $0 \leq \alpha \leq .8$, we must have $\frac{20}{20+74 \beta} \leq r \leq \frac{56}{56+34 \beta}, 0<\beta<.9$. The sure "Yes" case is approached as $\beta$ tends to zero.

\section{TWO VOTED SUBJECTS SYSTEMS}

In this case two voted subjects are considered. The model can be taken as follows 


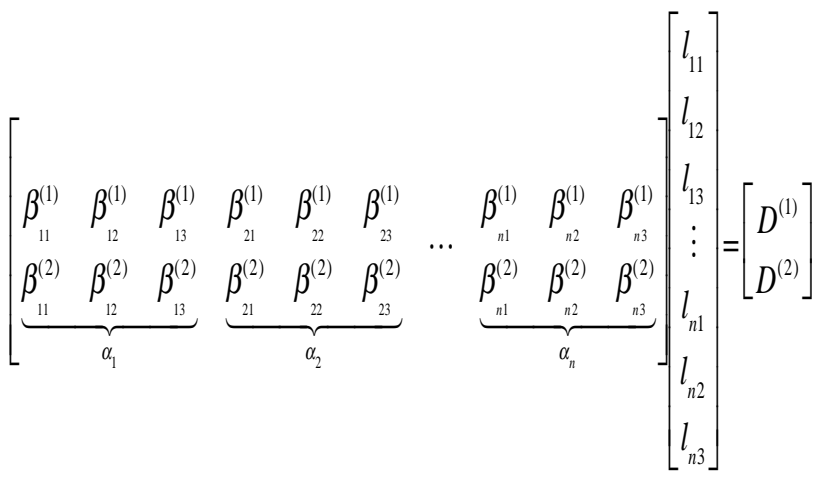

where $\mathrm{n}$ political groups are competing in deciding just two voted subjects. The population numbers $\beta_{i j}^{(m)}, j=1,2,3$ are independent than the population numbers $\beta_{i j}^{(k)}, j=1,2,3$ where $m \neq k$ since the population of the political group is divided independently for every voted subject. We can also assume that the policy is rigid for every group. Following the model, one can get the following "Yes" rates

$$
R_{y e s}^{(k)}=\frac{\sum_{i=1}^{n} \alpha_{i} \beta_{i 1}^{(k)} l_{i 1}}{D^{(k)}}, k=1,2
$$

where

$$
D^{(k)}=\sum_{i=1}^{n} \alpha_{i} \sum_{j=1}^{3} \beta_{i j}^{(k)} l_{i j}, k=1,2
$$

\section{Example-6.1}

Consider one political group with the following population weights

$\begin{array}{lll}\omega_{11}^{(1)}=.6 & \omega_{12}^{(1)}=.2 & \omega_{13}^{(1)}=.2 \\ \omega_{11}^{(2)}=.8 & \omega_{12}^{(2)}=.1 & \omega_{13}^{(2)}=.1\end{array}$

where one can notice the different weights w.r.t. each voted subject. Let us have the following "Yes" biased policy $l_{11}=1 \quad l_{12}=.5 \quad l_{13}=0$. The "Yes" rates are

$$
\begin{aligned}
R_{y e s}^{(1)} & =\frac{\beta_{11}^{(1)} l_{11}}{\beta_{11}^{(1)} l_{11}+\beta_{12}^{(1)} l_{12}} \\
& =.8571 \\
R_{y e s}^{(2)} & =\frac{\beta_{11}^{(2)} l_{11}}{\beta_{11}^{(2)} l_{11}+\beta_{12}^{(2)} l_{12}} \\
& =.9412
\end{aligned}
$$

One can notice the independency of computations. More complicated models can be described to model other complex cases.

\section{Example-6.2}

Consider three political groups as follows:

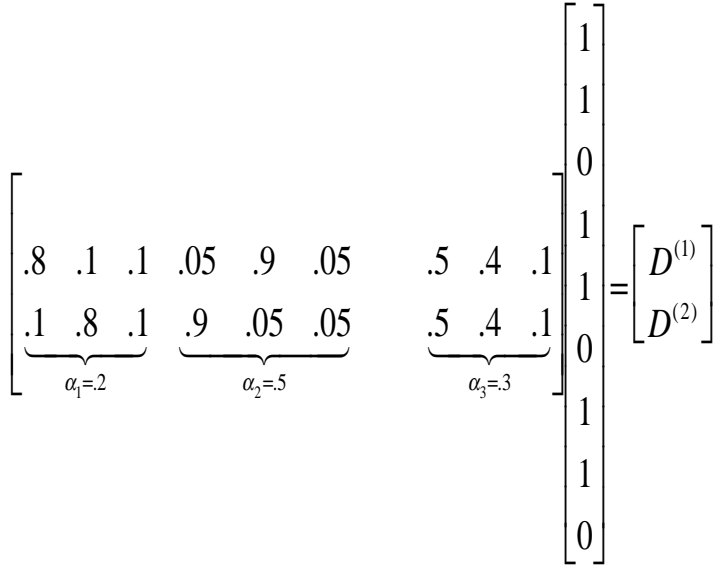

One can notice the fair voting and the varying decisions of the first group that it accepts the first subject and refuses the second. The second group has opposite opinions. The third party may have independent and fixed decisions against both voted subjects. Accordingly, the following "Yes" rates are computed:

$$
\begin{aligned}
R_{y e s}^{(1)} & =\frac{\beta_{11}^{(1)} l_{11} \alpha_{1}+\beta_{22}^{(1)} l_{22} \alpha_{2}+\beta_{33}^{(1)} l_{33} \alpha_{3}}{D^{(1)}} \\
& =36.22 \% \\
R_{y e s}^{(2)} & =\frac{\beta_{11}^{(2)} l_{11} \alpha_{1}+\beta_{22}^{(2)} l_{22} \alpha_{2}+\beta_{33}^{(2)} l_{33} \alpha_{3}}{D^{(2)}} \\
& =67.03 \%
\end{aligned}
$$

which are independent of each other. One can notice the success of the second group in both voted subjects may be because of the unpopularity of the first group.

We can extend the model to $\mathrm{m}$ voted subjects ( $\mathrm{m}$ winners case) and n political groups. tions.

We shall look for other opinions in the following sec-

\section{INVERSE PROBLEMS}

For the purpose of a future development, a group can study the voting system in reverse point of view. It can ask this question "What are the design population weights or the policy numbers which satisfy certain "Yes" rate criteria?. We are facing a rectangular system such that no unique values, if exist, are found. We are under the complete control of the different cases of the linear system of equations.

\section{Example-7.1}

Consider a ingle group single decision system and it is required to put the appropriate policy to satisfy the criteria $R_{y e s}^{(1)} \geq r$. In this case

$\frac{\omega_{11}^{(1)} l_{11}}{\omega_{11}^{(1)} l_{11}+\omega_{12}^{(1)} l_{12}+\omega_{13}^{(1)} l_{13}}>r$

Let the policy such that $l_{11}=x, l_{12}=y, l_{13}=0$. To satisfy the criteria, we should have 
$y \leq \frac{(1-r) \omega_{11}}{r \omega_{12}} x$

For $x \leq 1$, we get $y \leq \frac{(1-r) \omega_{11}}{r \omega_{12}}$. Fig. (15) illustrates the accepted region of y for different cases.

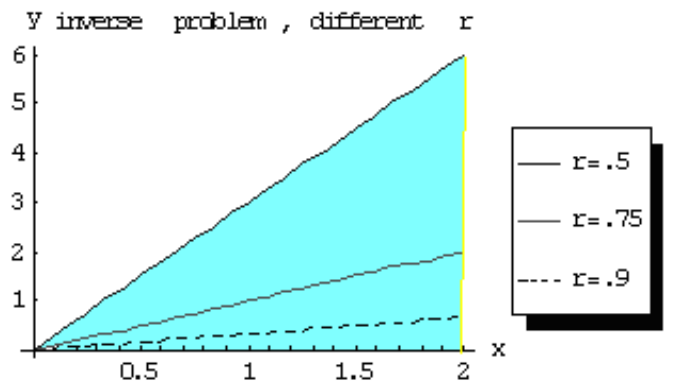

Fig. (15). The change of $\mathrm{y}$ with $\mathrm{x}$ for $\omega_{11}=.6, \omega_{12}=.2$ and different $\mathrm{r}$, (the shadow for the case of $\mathrm{r}=.5$ ).

One can use these charts at different population weights to satisfy a required criteria. The solution is not unique and a measure of selectivity may exist.

At known population weights, one can follow a different routine. Let us have $\omega_{11}=.5, \omega_{12}=.3, \omega_{13}=.2$. In this case, one gets the following

$\left[\begin{array}{lll}.5 & .3 & .2\end{array}\right]\left[\begin{array}{l}x \\ y \\ 0\end{array}\right]=[d]$

Solving this equation, we get infinite number of solutions as

$\left[\begin{array}{l}x \\ y\end{array}\right]=\left[\begin{array}{c}2 d \\ 0\end{array}\right]+c\left[\begin{array}{c}.6 \\ -1\end{array}\right]$

Now satisfying the criteria $R_{y e s}^{(1)} \geq r$, one can get

$d \geq \frac{-3(2 r+5) c}{10(5-3 r)}, c \in \mathbb{R}$.

Choosing $\mathrm{c}=-.8$ and $\mathrm{r}=.5$, one gets finally $y=.8, x \geq .3428$ from which we can choose $y=.8$ and $x=1$.

This routine depends greatly on having a solution to the given system of equations.

\section{Example-7.2}

Let us have two groups and a single decision.

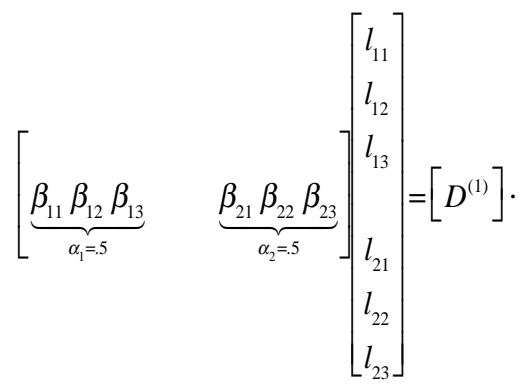

Required to satisfy $R_{y e s}^{(1)} \geq r$.Let $\quad\left(l_{11}=x_{1}, l_{21}=x_{2}\right.$, $\left.l_{12}=y_{1}, l_{22}=y_{2}, l_{13}=l_{23}=0\right)$. This leads to the following

$\frac{\omega_{11}^{(1)} x_{1}+\omega_{21}^{(1)} x_{2}}{\omega_{11}^{(1)} x_{1}+\omega_{21}^{(1)} x_{2}+\omega_{12}^{(1)} y_{1}+\omega_{22}^{(1)} y_{2}} \geq r$

Let $x_{1}=x_{2}=1$, then we get

$r \omega_{12}^{(1)} y_{1}+r \omega_{22}^{(1)} y_{2} \leq(1-r)\left(\omega_{11}^{(1)}+\omega_{21}^{(1)}\right)$,

or any other inequalities at different cases. The graph of the inequality can illustrate the feasible region of $y_{1}$ and $y_{2}$.For example, taking the values

$\begin{array}{lll}\omega_{11}^{(1)}=.5 & \omega_{12}^{(1)}=.3 & \omega_{13}^{(1)}=.2 \\ \omega_{11}^{(2)}=.6 & \omega_{12}^{(2)}=.2 & \omega_{13}^{(2)}=.2\end{array}$

then the following inequality is obtained:

$3 y_{1}+2 y_{2} \leq 2.75$.

Fig. (16) illustrates the accepted region of selectivity:

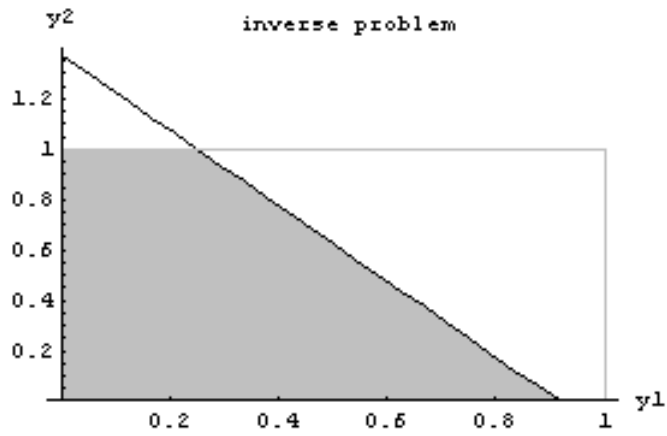

Fig. (16). The feasible region of $y_{1}$ and $y_{2}$.

One can design the system according to the feasible region got in the figure.

\section{RANDOM MODELS}

In this case, one or more of the variables and parameters of the voting system may follow random or stochastic behavior. Let us illustrate the random behavior through a one group-one decision model. For simplicity, let $\beta_{13}=0$ then $\beta_{11}+\beta_{12}=N$. Dividing by the total allowable population $N$, then $x+y=1$. Let us assume that the "Yes" population weight $x$ is random and can have three possibilities.

$x \sim u(0,1)$ : uniform distribution.

Since $y=1-x$, the "No" population weight should be uniform too, i.e. $y \sim u(0,1)$. The "Yes" rate takes the following formula

$R_{y e s}=\frac{l_{11} x}{\left(l_{11}-l_{12}\right) x+l_{12}}$. 
We have two cases.

i) Fair voting

In this case, $l_{11}=l_{12}=1, l_{13}=0$ which leads to $R_{\text {yes }}=x$. This means that the "Yes" rate is uniformly distributed too. Then the following basic probability relations are true

$$
P\left(R_{\text {yes }}>.5\right)=P\left(R_{\text {yes }}<.5\right)=.5 \text {. }
$$

\section{ii) Unfair voting}

Let us examine the "No rejection" and "Yes additions" policies and show the effect of these policies on the probability distribution function (p.d.f.) of the "Yes" rate.

ii-1) "Yes" increase policy

In this case, $l_{11}=1+\alpha, l_{12}=1$ which leads to

$R_{y e s}=\frac{(1+\alpha) x}{1+\alpha x}, \alpha>0$.

One can notice that in general

$$
\begin{aligned}
\left.R_{\text {yes }}\right|_{\text {unfair }} & =\frac{(1+\alpha) x}{1+\alpha x}, 0>x>1 \\
& >\left.R_{\text {yes }}\right|_{\text {fair }}
\end{aligned}
$$

Using the theory of random variable transformation [10], we can obtain the following probability density function (p.d.f.) of the "Yes" rate

$f_{R}(r)=\frac{(1+\alpha)}{(1+\alpha(1-r))^{2}}, 0 \leq r \leq 1$.

Fig. (17) illustrates the deformations of the pd.f. of the "Yes" rate around uniformity.

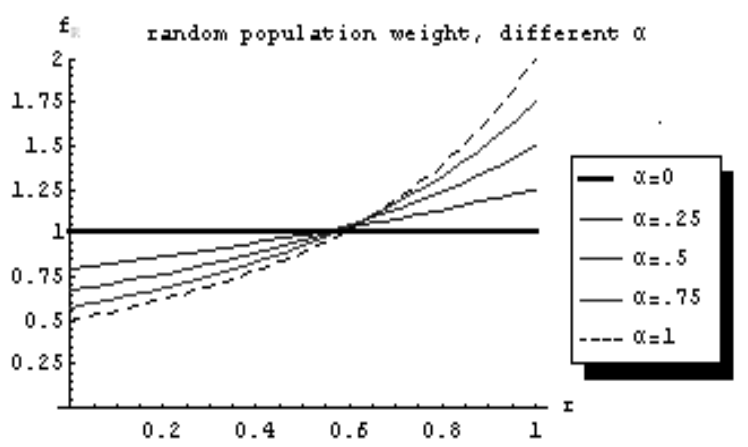

Fig. (17). The deviation of the p.d.f. of the "Yes" rate around uniformity ( $\alpha=0)$.

One can notice that the higher values of $\alpha$ the higher deviations around uniformity. We can compute a significant probability as follows:

$$
p\left(R_{y e s}>q\right)=\frac{(1+\alpha)(1-q)}{1+\alpha(1-q)}, \alpha>0, q \geq .5 .
$$

Fig. (18) illustrates the change of the probability with $\alpha$.

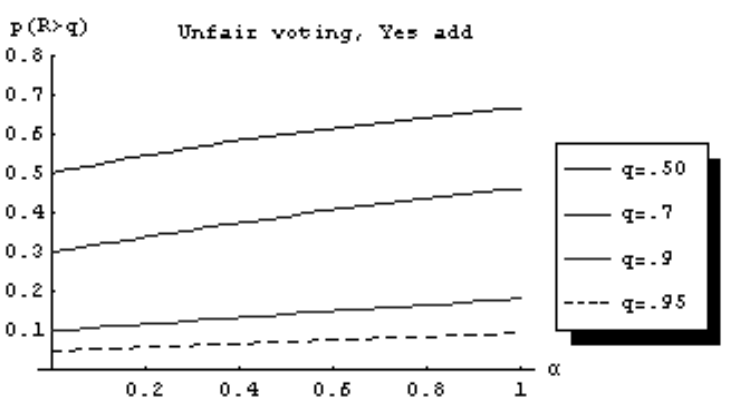

Fig. (18). The change of $p\left(R_{y e s}>q\right)$ with $\alpha$.

One can notice the continuous increase of the probability with $\alpha$ and its continuous decrease with the increase of q. Theoretically, the probability approaches unity when $\alpha$ approaches $\infty$.

ii-2) "No" rejection policy

In this case, $l_{11}=1, l_{12}=\beta$ which leads to

$R_{y e s}=\frac{x}{\beta+(1-\beta) x}, 0>\beta>1$.

One can also notice that

$$
\begin{aligned}
R_{\text {yes }\left.\right|_{\text {unfair }}} & =\frac{x}{\beta+(1-\beta) x}, 0>x>1 \\
& >\left.R_{\text {yes }}\right|_{\text {fair }}
\end{aligned}
$$

Using the theory of random variable transformation, we can obtain the following p.d.f. of the "Yes" rate

$$
f_{R}(r)=\frac{\beta}{(1-(1-\beta) r)^{2}}, 0 \leq r \leq 1 .
$$

Fig. (19) illustrates the deformations of the pd.f. of the "Yes" rate around uniformity.

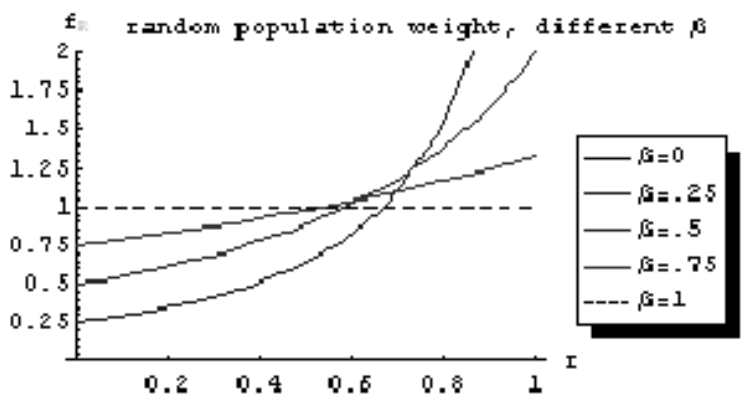

Fig. (19). The deviation of the p.d.f. of the "Yes" rate around uniformity $(\beta=1)$.

One can notice that the higher values of $\beta$ the lower deviations around uniformity. We can compute a significant probability as follows: 


$$
p\left(R_{y e s}>q\right)=\frac{(1-q)}{1-(1-\beta) q}, q \geq .5
$$

Fig. (20) illustrates the change of the probability with $\beta$.

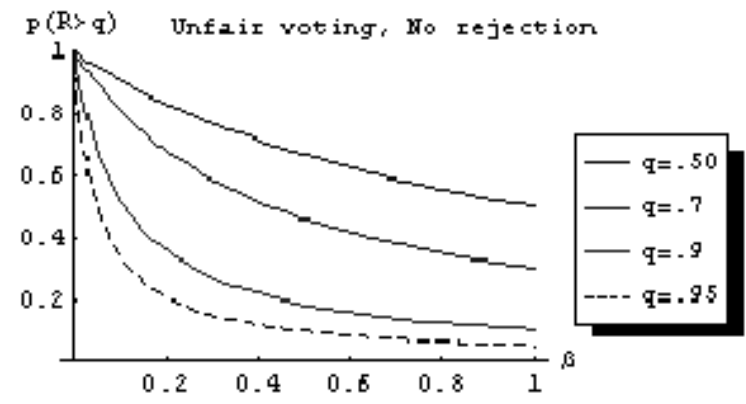

Fig. (20). The change of $p\left(R_{\text {yes }}>q\right)$ with $\beta$.

One can notice the continuous decrease of the probability with the increase of $\beta$ and its continuous decrease with the increase of q. Theoretically, the probability approaches unity when $\beta$ approaches 1 .

\section{ii-3) Combination policy}

Let us combine the two previous policies. The following results hold

$$
\begin{aligned}
& R_{y e s}=\frac{(1+\alpha) x}{\beta+[(1+\alpha)-\beta] x}, \\
& f_{R}(r)=\frac{(1+\alpha) \beta}{[(1+\alpha)-(1+\alpha-\beta) r]^{2}}, \\
& p\left(R_{y e s}>.5\right)=\frac{(1+\alpha)}{1+\alpha+\beta} .
\end{aligned}
$$

One can notice that the case ii-1) can be reached at $\beta=1$ while the case ii-2) is reached when $\alpha=0$. An important question arises in this case, what are the values of $\alpha$ and $\beta$ to reach a maximum value for $p\left(R_{\text {yes }}>.5\right)$. This can be simply got using optimization.

\section{$x \sim \operatorname{fall}(\lambda):$ Fall distribution}

If the probability of the population weight of "Yes" voters is high for low value, case of fall distribution which illustrates that the political group suffers, the following fall distribution can be suggested

$$
f_{x}(x ; \lambda)=\frac{A(1-x)}{1+\lambda x}, \lambda>0
$$

or $x \sim \operatorname{fall}(\lambda)$ where

$$
A=\frac{\lambda^{2}}{(1+\lambda) \ln (1+\lambda)-\lambda} \text {. }
$$

Fig. (21) illustrates the distribution shape.

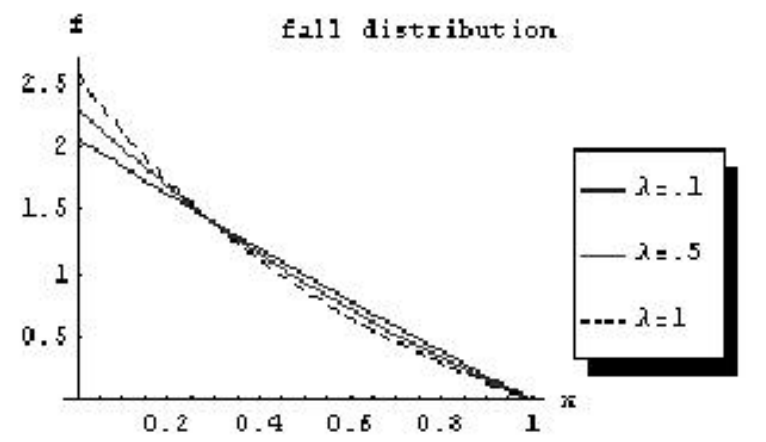

Fig. (21). the change of the fall distribution with the parameter $\lambda$.

The probability that $\mathrm{x}$ is at least $\alpha$ can be computed as $p(x \geq \alpha)=1+\frac{A \alpha}{\lambda}-A\left(\frac{1}{\lambda}+\frac{1}{\lambda^{2}}\right) \ln (1+\alpha \lambda)$.

Now the Yes ratio can be discussed as before as follows:

i) Fair voting

In this case, $R_{y e s}=x$ which means that the Yes ratio also belongs to the fall distribution. The probability that the Yes ratio is at least $50 \%$ can be evaluated as

$$
p\left(R_{y e s} \geq .5\right)=1+\frac{A}{2 \lambda}-A\left(\frac{1}{\lambda}+\frac{1}{\lambda^{2}}\right) \ln \left(1+\frac{\lambda}{2}\right) .
$$

Fig. (22) illustrates the change of the probability of the Yes ratio being at leas .5 with $\lambda$.

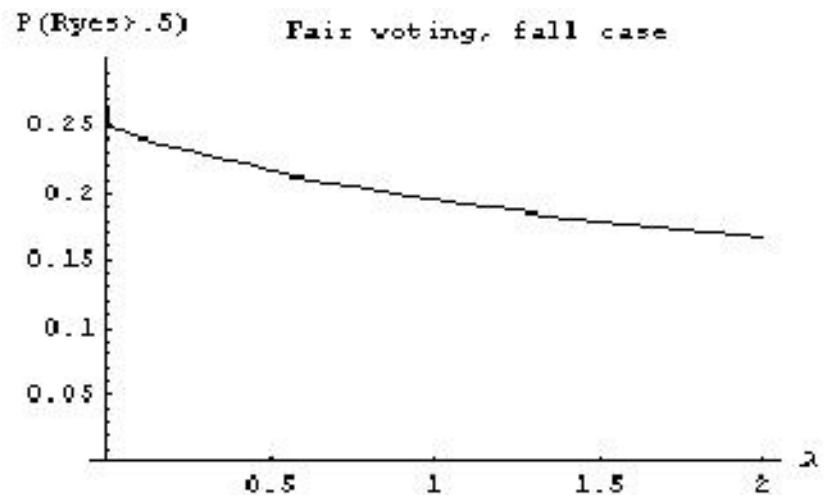

Fig. (22). The change of $p\left(R_{y e s} \geq .5\right)$ with $\lambda$.

One ca notice that the Yes rate is also suffering as the political group.

\section{ii) Unfair voting}

With the aid of random variable transformation, the p.d.f. of the Yes ratio under Yes increase policy, $l_{11}=1+\gamma, l_{12}=1$, can be evaluated as

$$
f_{R}(r)=\frac{A(1+\gamma)^{2}(1-r)}{[1+\gamma+(\lambda-\gamma) r][1+\gamma(1-r)]^{2}}, \gamma>0,0 \leq r \leq 1
$$

Fig. (23) illustrate the shape of the p.d.f. 


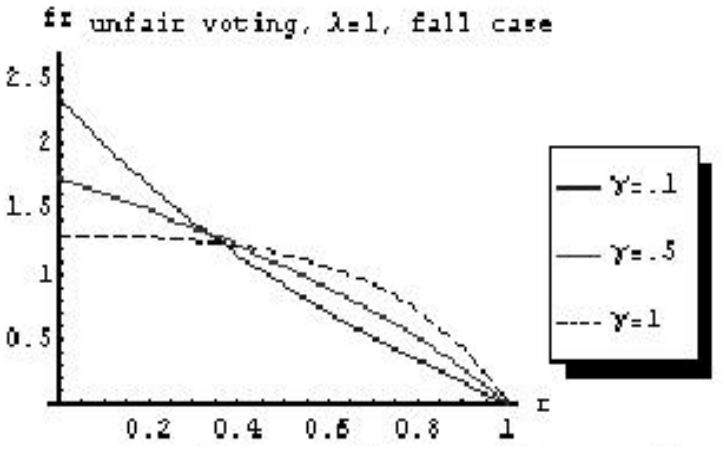

Fig. (23). The change of the Yes ratio p.d.f. with r.

The probability that the Yes ratio is at least $\theta$ can be evaluated as

$p\left(R_{y e s} \geq \theta\right)=\frac{A}{\lambda^{2}}\left[(1+\lambda) \ln \frac{(1+\lambda)(1+\gamma(1-\theta))}{1+\gamma+(\lambda-\gamma) \theta}-\frac{\lambda(1+\gamma)(1-\theta)}{1+\gamma(1-\theta)}\right]$

Fig. (24) illustrates this probability at $\theta=.5$.

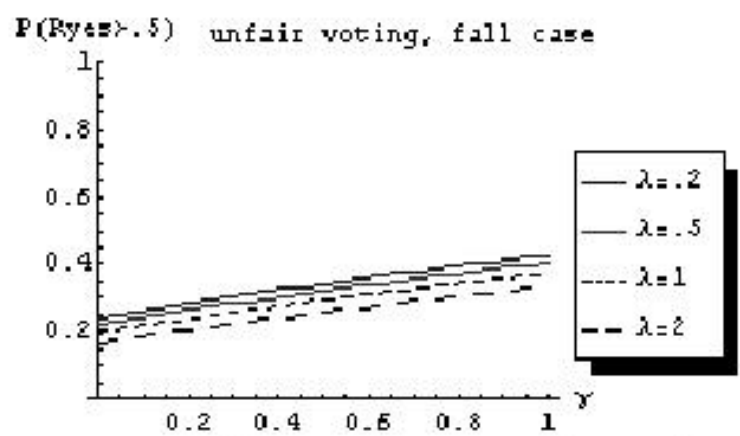

Fig. (24). The change of $p\left(R_{y e s} \geq .5\right)$ at different $\lambda$.

The low chance of the suffering political group is completely noticed even when taking unfair policies.

$x \sim \operatorname{rise}(\lambda):$ Rise distribution

If the probability of the population weight of "Yes" voters is high for high value, case of rise distribution which illustrates that the political group is succeeding, the following rise distribution can be suggested

$$
f_{x}(x ; \rho)=\frac{A x}{1+\rho(1-x)}, \rho>0
$$

or $x \sim \operatorname{rise}(\rho)$ where

$$
A=\frac{\rho^{2}}{(1+\rho) \ln (1+\rho)-\rho} .
$$

Fig. (25) illustrates the distribution shape

The probability that $\mathrm{x}$ is at least $\alpha$ can be computed as $p(x \geq \alpha)=1+\frac{A \alpha}{\lambda}+A\left(\frac{1}{\lambda}+\frac{1}{\lambda^{2}}\right) \ln \left(\frac{1+\lambda(1-\alpha)}{1+\lambda}\right)$.

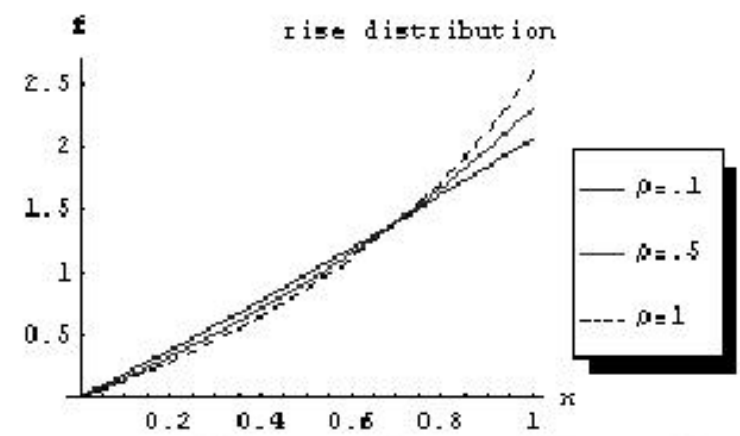

Fig. (25). The change of the rise distribution with the parameter $\rho$.

Now the Yes ratio can be discussed as before as follows:

i) Fair voting

In this case, $R_{y e s}=x$ which means that the Yes ratio also belongs to the rise distribution.

The probability that the Yes ratio is at least $50 \%$ can be evaluated as

$p\left(R_{y e s} \geq .5\right)=1+\frac{A}{2 \rho}+A\left(\frac{1}{\rho}+\frac{1}{\rho^{2}}\right) \ln \left(\frac{1+\frac{\rho}{2}}{1+\rho}\right)$.

Fig. (26) illustrates the change of the probability of the Yes ratio being at leas .5 with $\rho$.

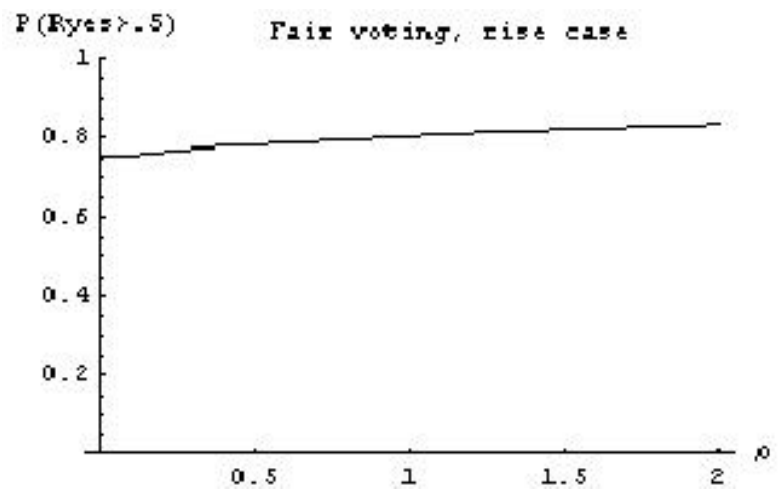

Fig. (26). The change of $p\left(R_{y e s} \geq .5\right)$ with $\rho$.

One can notice the success of this rise group.

ii) Unfair voting

With the aid of random variable transformation, the p.d.f. of the Yes ratio under Yes increase policy, $l_{11}=1+\gamma, l_{12}=1$, can be evaluated as

$$
f_{R}(r)=\frac{A(1+\gamma) r}{[1+\gamma(1-r)+\rho(1+\gamma)(1-r)][1+\gamma(1-r)]^{2}}, \gamma>0,0 \leq r \leq 1
$$

Fig. (27) illustrate the shape of the p.d.f.

The probability that the Yes ratio is at least $\theta$ can be evaluated as 


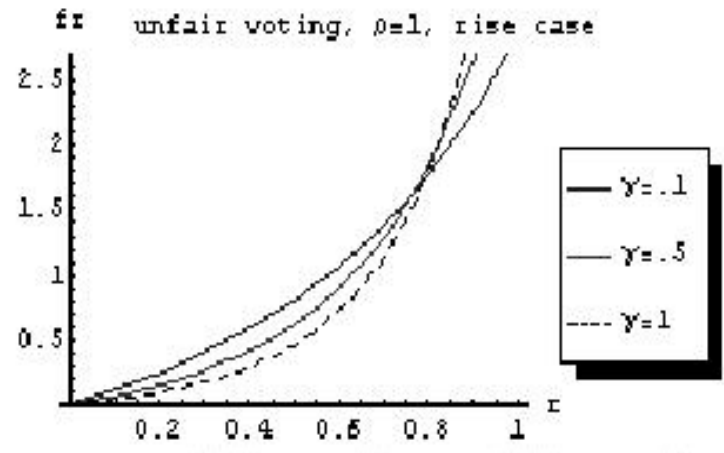

Fig. (27). The change of the Yes ratio p.d.f. with r.

$$
p\left(R_{y e s} \geq \theta\right)=\frac{A}{\rho^{2}}\left[\begin{array}{l}
(1+\rho) \ln \left((1+\rho)(1+\gamma)-\theta(\gamma+\rho(1+\gamma))-\frac{\lambda(1+\gamma)(1-\theta)}{1+\gamma(1-\theta)}\right. \\
+\frac{\rho}{\gamma} \ln (1+\gamma(1-\theta))-\frac{\gamma(1+\rho)+\rho}{\gamma} \ln (1+\gamma(1-\theta))
\end{array}\right] .
$$

Fig. (28) illustrates this probability at $\theta=.5$.

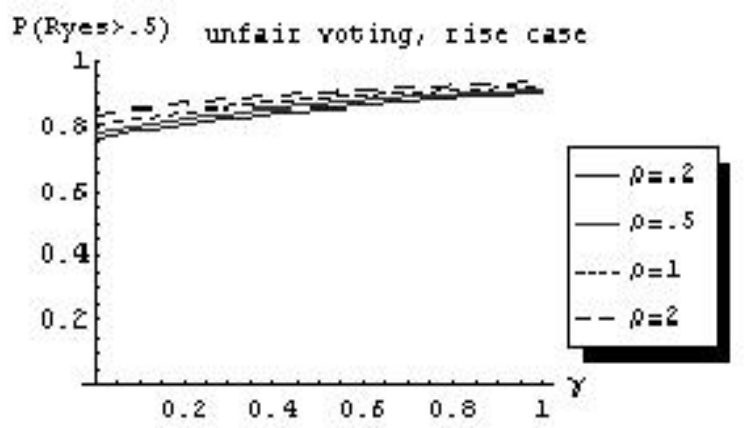

One can notice that the Yes increase policy has increased the success of this succeeding group.

\section{CONCLUSIONS}

In this paper, the voting systems are introduced as linear transformation models. The general system of $n$ political groups and $\mathrm{m}$ voted subjects is introduced using the compartmental populations w.r.t. "Yes", "No" and "Neutral" votes of each political group and reaction policy parameters of the voting responsible board. Different systems have been discussed including "fair" and "unfair" voting systems with the "No" rejection and "Yes" increase policies in deterministic and a simple stochastic models. The inverse problem is also introduced. I believe that interesting more general studies can be achieved using this concept.

\section{REFERENCES}

[1] Arrow K. Social choice and individual values. $2^{\text {nd }}$ ed. New Haven: Yale University Press 1963.

[2] Colomer JM, Ed. Handbook of electoral system choice. London NY: Palgrave-Macmillan 2004.

[3] Farrell DM. Elecoral systems: a comparative introduction. NY, USA: Martin's Press 2001.

[4] Eiassan C, Zuguete A. An electronic voting system supporting vote weights. Inter Res 2006; 16(5): 507-18.

[5] Brams SJ, Sanver MR. Critical strategies under approval voting : who gets ruled in and ruled out. Electoral Stud 2006; 25(2): 287 305.

[6] Rosema M. Partisanship, candidate evaluations and prospective voting. Electoral Stud 2006; 25(3): 467-88.

[7] Xie M, Pham H. Modeling the reliability of threshold weighted voting systems. Reliab Eng Syst Saf 2005; 87(1): 53-63.

[8] Brg S. Indirect voting systems: Banzhaf numbers, majority functions and collective completeness. Eur J Polit Econ 1997; 13(3): 557-73.

[9] Merlin V, Valognes F. The impact of indifferent voters on the likelihood of some voting paradoxes. Mathe Soc Sci 2004; 48(3): 343-61.

[10] El-Tawil M. Probabilities: theory and applications. $2^{\text {nd }}$ ed. Cairo : Dar Nashr El-Gmaat 1997.

Fig. (28). The change of $p\left(R_{y e s} \geq .5\right)$ at different $\rho$.

(c) M.A. El-Tawil; Licensee Bentham Open.

This is an open access article licensed under the terms of the Creative Commons Attribution Non-Commercial License (http://creativecommons.org/licenses/by-nc/3.0/) which permits unrestricted, non-commercial use, distribution and reproduction in any medium, provided the work is properly cited. 